\title{
LONG-TERM TRYPTOPHAN SUPPLEMENTATION DECREASED THE WELFARE AND INNATE IMMUNE STATUS OF PIKEPERCH JUVENILES
}

\author{
Syaghalirwa N.M. Mandiki ${ }^{1 * \$}$, Baptiste Redivo ${ }^{1 *}$, Sébastien Baekelandt ${ }^{1 *}$, Jessica \\ Douxfils $^{1 *}$, Ivar Lund ${ }^{2 *}$, Erik Höglund ${ }^{2 *}$, Patrick Kestemont ${ }^{1 *}$, \\ ${ }^{1}$ Research Unit of Environmental and Evolutionary Biology, University of Namur, Rue de \\ Bruxelles 61, 5000 Namur-Belgium.
}

${ }^{2}$ Technical University of Denmark, Section Aquaculture, Nordsфen Forskerpark/The North Sea Science Park P.O. Box 101, Denmark.

\begin{abstract}
It has been demonstrated that short-term supplementation of L-tryptophan (TRP) can mitigate the primary neuroendocrine response to stress in some fish species, but such stress reduction was reported to be both dose- and context-dependent (Basic et al, 2013; Machado et al, 2015). So, the TRP responses may be species related or depend on the stress levels experienced by the fish. Since percid fish were reported to be more stress responsiveness than common aquaculture species such as rainbow trout (Jentoft et al, 2005), this study aimed (1) to determine to what extent a long-term dietary TRP mitigate the physiological response of pikeperch (Sander lucioperca) to emersion stress, and (2) to characterize the related immune status. Pikeperch juveniles of $10 \mathrm{~g}$ received four experimental diets: $(\mathrm{CT})=$ control groups without any stress and any feed TRP supplement, (CTs) = control groups submitted to emersion stress but without any feed TRP supplement, 3TRPs = groups receiving 3-time TRP diet and submitted to emersion stress, 6TRPs = groups receiving 6-time TRP diet and submitted to emersion stress. Various organs were sampled on D7, D37 and D91 of TRP supplementation for evaluation of physiological and immune responses; samplings were done one hour after the emersion challenge stress. Specific growth rate (SGR) as well as food conversion rate (FCR) were also checked. Emersion stress induced a significant increase in plasma cortisol both after a single stress or repeated stress challenges. Dietary TRP significantly decreased cortisol levels in a dose related manner both after a single or repeated stress; but plasma glucose level was only affected after a single stress but not after long-term feeding. The two stress indicators measured showed that pikeperch displayed higher stress responsiveness as already demonstrated for European perch in comparison to salmonids (Jentoft et al, 2005). The reduction in physiological stress status by dietary TRP was associated to a significant decrease in plasma lysosomal activity, especially on D91; indicating negative interaction with the innate immune pathways. Growth rate was slowed by the long- term dietary TRP in association to an increase in FCR values. The available results indicate that long-term TRP supplementation has negative impact on the overall welfare status of pikeperch. Other analyses are ongoing and more results concerning key- brain neurotransmitters such as serotonin, dopamine and their metabolites concentrations as well as on the expressions of key-immune genes (C3-1, TNF- $\alpha$, IL-1 $\beta$, etc) will be discussed.
\end{abstract}

\section{KEYWORDS}

L-tryptophan, stress responsiveness, immunity, welfare status, pikeperch 
*These authors have contributed equally to this work.

${ }^{\S}$ Corresponding author. Tel.: +3281724284; Fax: +3281724362.

E-mail address: robert.mandiki@unamur.be 